\title{
STANCU TYPE GENERALIZATION OF THE $q$-FAVARD-SZÀSZ OPERATORS
}

\author{
ALİ KARAİSA, DURHASAN TURGUT TOLLU AND YASIN ASAR
}

\begin{abstract}
In this paper, we introduce a Stancu type generalization of the $q$-Favard-Szàsz operators, estimate
\end{abstract} the rates of statistical convergence and study the local approximation properties of these operators.

\section{INTRODUCTION}

In [10], Jakimovski and Leviatan introduced a Favard Szàsz type operator, by using Appell polynomials $p_{k}(x), k \geqslant 0$, defined by

$$
g(u) e^{-u x}=\sum_{k=0}^{\infty} p_{k}(x) u^{k}
$$

where $g(z)=\sum_{n=0}^{\infty} a_{n} z^{n}$ is an analytic function in the disc $|z|<R, R>1$ and $g(1) \neq 0$, and they established several approximation properties of these operators. Ciupa [8], by defined the following operators

$$
P_{n, t}(f ; x)=\frac{e^{-n x}}{g(1)} \sum_{k=0}^{\infty} p_{k}(n t) f\left(x+\frac{k}{n}\right)
$$

and investigated the approximation properties and the rate of convergence of these operators via the modulus of continuity.

In [6], Atakut and Büyükyazıcı studied some approximation properties of the operators

$$
P_{n, t}^{\alpha, \beta}(f ; x)=\frac{e^{-n x}}{g(1)} \sum_{k=0}^{\infty} p_{k}(n t) f\left(x+\frac{k+\alpha}{n+\beta}\right)
$$

where $p_{k}(n t)$ is an Appell polynomial in $n t$ which is a Stancu type generalization of the classical Favard-Szàsz operators. Moreover, In [5], the same authors established the approximation properties of the operators

$$
L_{n}(f ; q, x)=\frac{E^{-[n]_{q} t}}{A(1)} \sum_{k=0}^{\infty} \frac{P_{k}\left(q ;[n]_{q} t\right)}{[k]_{q} !} f\left(x+\frac{[k]_{q}}{[n]_{q}}\right),
$$

which is a $q$-analogue of the classical Favard-Szàsz operators related to the $q$-Appell polinomials. They also estimated the rate of convergence of these operators.

Now, let us define Stancu type generalization of the q-Favard-Szàsz operators as follows:

$$
T_{n, t}^{\alpha, \beta}(f ; q ; x)=\frac{E_{q}^{-[n]_{q} t}}{A(1)} \sum_{k=0}^{\infty} \frac{P_{k}\left(q ;[n]_{q} t\right)}{[k]_{q} !} f\left(x+\frac{[k]_{q}+\alpha}{[n]_{q}+\beta}\right),
$$

where $\left\{P_{k}(q ; .)\right\}_{k \geqslant 0}$ is a $q$-Appell polynomial set which is generated by

$$
A(u) e_{q}^{[n]_{q} t u}=\sum_{k=0}^{\infty} \frac{P_{k}\left(q ;[n]_{q} t\right) u^{k}}{[k]_{q} !}
$$

and $A(t)$ is defined by

$$
A(u)=\sum_{k=0}^{\infty} a_{k} u^{k}
$$

In this work, we investigate a Korovkin theorem and the rate of statistical convergence by using modulus of continuity of (1.1). We also obtain some local approximation results of these new operators.

2010 Mathematics Subject Classification. 40A05, 40A25, 41A36.

Key words and phrases. Favard-Szàsz operators, q-integers, Modulus of smoothness, Statistical convergence, Rate of convergence. 
Let us recall some definitions and notations regarding the concept of $q-$ calculus. Further results can be found in [7]. In the sequel, $q$ is a real number satisfying $0<q<1$. For $n \in \mathbb{N}$, the $q$-integer $[n]_{q}$ is defined by

$$
[n]_{q}:=\frac{1-q^{n}}{1-q}
$$

and the $q$-factorial $[n]_{q}$ ! is defined as following

$$
[n]_{q} !:= \begin{cases}{[n]_{q}[n-1]_{q} \cdots[1]_{q},} & n \in \mathbb{N} \\ 1, & n=0\end{cases}
$$

The $q$-binomial coefficients are given by

$$
\left[\begin{array}{l}
n \\
k
\end{array}\right]_{q}=\frac{[n]_{q} !}{[k]_{q} ![n-k]_{q} !}, 0 \leqslant k \leqslant n .
$$

The $q$-derivative $D_{q} f$ of a function $f$ is defined by

$$
\left(D_{q} f\right)(x)=\frac{f(x)-f(q x)}{(1-q) x}, x \neq 0 .
$$

Also, if there exists $\frac{d f}{d x}(0)$, then $\left(D_{q} f\right)(0)=\frac{d f}{d x}(0)$. The following q-derivatives of the product of the functions $f(x)$ and $g(x)$ are equivalent:

$$
D_{q}(f(x) g(x))=f(q x) D_{q} g(x)+g(x) D_{q} f(x)
$$

and

$$
D_{q}(f(x) g(x))=f(x) D_{q} g(x)+g(q x) D_{q} f(x) .
$$

The $q$-analogues of the exponential function are given by

$$
e_{q}^{x}=\sum_{n=0}^{\infty} \frac{x^{n}}{[n]_{q} !}
$$

and

$$
E_{q}^{x}=\sum_{n=0}^{\infty} q^{\frac{n(n-1)}{2}} \frac{x^{n}}{[n]_{q} !} .
$$

The exponential functions have the following properties:

$$
D_{q}\left(e_{q}^{a x}\right)=a e_{q}^{a x}, D_{q}\left(E_{q}^{a x}\right)=a E_{q}^{a q x}, e_{q}^{x} E_{q}^{-x}=E_{q}^{x} e_{q}^{-x}=1 .
$$

\section{STATisticAl APPROXIMATION PROPERTIES}

Before proceeding further, let us give basic definition and notation on the concept of the statistical convergence which was introduced by Fast [1]. Let $K$ be a subset of $\mathbb{N}$, the set of natural numbers. Then, $K_{n}=\{k \leqslant n: k \in K\}$. The natural density of $K$ is defined by $\delta(K)=\lim _{n} \frac{1}{n}\left|K_{n}\right|$ provided that the limit exists, where $\left|K_{n}\right|$ denotes the cardinality of the set $K_{n}$. A sequence $x=\left(x_{k}\right)$ is called statistically convergent to the number $\ell \in \mathbb{R}$, denoted by $s t-\lim x=\ell$. For each $\epsilon>0$, the set $K_{\varepsilon}=\left\{k \in \mathbb{N}:\left|x_{k}-\ell\right| \geqslant \epsilon\right\}$ has a natural density zero, that is

$$
\lim _{n \rightarrow \infty} \frac{1}{n}\left|\left\{k \leqslant n:\left|x_{k}-\ell\right| \geqslant \epsilon\right\}\right|=0 .
$$

It is well know that every statistically convergence sequence is ordinary convergent, but the converse is not true.

The concept of statistical convergence was firstly used in approximation theory by Gadjiev and Orhan [12]. They proved the Bohman-Korovkin type approximation theorem for statistical convergence. For further information related to the statistical approximation of the operators, the followings are remarkable among others: [13 17].

Now, we may begin the following lemma which is needed proving our main result. 
Lemma 2.1. For $n \in \mathbb{N}, x \in[0, \infty)$ and $0<q<1$, we have

$$
\begin{aligned}
T_{n, t}^{\alpha, \beta}\left(e_{0}(t) ; q ; x\right)= & 1, \\
T_{n, t}^{\alpha, \beta}\left(e_{1}(t) ; q ; x\right)= & x+\frac{[n]_{q} t}{[n]_{q}+\beta}+\frac{\alpha}{[n]_{q}+\beta}+\frac{R D_{q}(A(1))}{[n]_{q}+\beta} \\
T_{n, t}^{\alpha, \beta}\left(e_{2}(t) ; q ; x\right)= & \left(x+\frac{[n]_{q} t}{[n]_{q}+\beta}\right)^{2}+\frac{2 x\left[\alpha+R D_{q}(A(1))\right]}{[n]_{q}+\beta} \\
& +\frac{\alpha^{2}+2 R \alpha D_{q}\left(A(1)+D_{q}^{2}(A(1))\right.}{\left([n]_{q}+\beta\right)^{2}}+\frac{[n]_{q} t\left[2 \alpha+D_{q}(A(1))\right]}{\left([n]_{q}+\beta\right)^{2}}+\frac{q[n]_{q} t}{\left([n]_{q}+\beta\right)^{2}},
\end{aligned}
$$

where $R=\frac{\left.e_{q}^{q[n]}\right]_{q}^{t} E_{q}^{-[n]} q^{t}}{A(1)}$, and $e_{v}(t)=(x+t)^{v}, v=0,1,2$.

Proof. If we consider (1.2), we can show the following

$$
\begin{gathered}
\sum_{k=0}^{\infty} \frac{p_{k}\left(q ;[n]_{q} t\right)}{[k]_{q} !}=A(1) e_{q}^{[n]_{q} t}, \\
\sum_{k=0}^{\infty} \frac{p_{k}\left(q ;[n]_{q} t\right)}{[k]_{q} !}[k]_{q}=A(1)[n]_{q} t e_{q}^{[n]_{q} t}+e_{q}^{q[n]_{q} t} D_{q} A(1)
\end{gathered}
$$

and

$$
\sum_{k=0}^{\infty} \frac{p_{k}\left(q ;[n]_{q} t\right)}{[k]_{q} !}[k]_{q}^{2}=D_{q}^{2}(A(1)) e_{q}^{[n]_{q} t}+D_{q}(A(1))[n]_{q} t e_{q}^{[n]_{q} t}+[n]_{q} t D_{q} A(q) e_{q}^{q[n]_{q} t}+A(1)[n]_{q}^{2} t^{2} e_{q}^{[n]_{q} t}
$$

By using the relations (2.4)-(2.6), from (1.1), we obtain the results

$$
\begin{aligned}
& T_{n, t}^{\alpha, \beta}\left(e_{0}(t) ; q ; x\right)=\frac{E_{q}^{-[n]_{q} t}}{A(1)} \sum_{k=0}^{\infty} \frac{p_{k}\left(q ;[n]_{q} t\right)}{[k]_{q} !}=\frac{E_{q}^{-[n]_{q} t}}{A(1)} A(1) e_{q}^{[n]_{q} t}=1, \\
& T_{n, t}^{\alpha, \beta}\left(e_{1}(t) ; q ; x\right)= \frac{E_{q}^{-[n]_{q} t}}{A(1)} \sum_{k=0}^{\infty} \frac{p_{k}\left(q ;[n]_{q} t\right)}{[k]_{q} !}\left(x+\frac{[k]_{q}+\alpha}{[n]_{q}+\beta}\right) \\
&=x+\frac{\alpha E_{q}^{-[n]_{q} t}}{A(1)\left([n]_{q}+\beta\right)} \sum_{k=0}^{\infty} \frac{p_{k}\left(q ;[n]_{q} t\right)}{[k]_{q} !}+\frac{E_{q}^{-[n]_{q} t}}{A(1)\left([n]_{q}+\beta\right)} \sum_{k=0}^{\infty} \frac{p_{k}\left(q ;[n]_{q} t\right)}{[k]_{q} !}[k]_{q} \\
&=x+\frac{\alpha}{[n]_{q}+\beta}+\frac{E_{q}^{-[n]_{q} t}}{A(1)\left([n]_{q}+\beta\right)}\left(A(1)[n]_{q} t e_{q}^{[n]_{q} t}+e_{q}^{q[n]_{q} t} D_{q} A(1)\right) \\
&=x+\frac{[n]_{q} t}{[n]_{q}+\beta}+\frac{\alpha}{[n]_{q}+\beta}+\frac{D_{q}(A(1)) R}{[n]_{q}+\beta}
\end{aligned}
$$


and

$$
\begin{aligned}
T_{n, t}^{\alpha, \beta}\left(e_{2}(t) ; q ; x\right)= & \frac{E_{q}^{-[n]_{q} t}}{A(1)} \sum_{k=0}^{\infty} \frac{p_{k}\left(q ;[n]_{q} t\right)}{[k]_{q} !}\left(x+\frac{[k]_{q}+\alpha}{[n]_{q}+\beta}\right)^{2} \\
= & x^{2}+\frac{2 \alpha x}{[n]_{q}+\beta}+\frac{\alpha^{2}}{\left([n]_{q}+\beta\right)^{2}}+\frac{2 x E_{q}^{-[n]_{q} t}}{A(1)\left([n]_{q}+\beta\right)} \sum_{k=0}^{\infty} \frac{p_{k}\left(q ;[n]_{q} t\right)}{[k]_{q} !}[k]_{q} \\
& +\frac{2 \alpha E_{q}^{-[n]_{q} t}}{A(1)\left([n]_{q}+\beta\right)^{2}} \sum_{k=0}^{\infty} \frac{p_{k}\left(q ;[n]_{q} t\right)}{[k]_{q} !}[k]_{q} \\
& +\frac{E_{q}^{-[n]_{q} t}}{A(1)\left([n]_{q}+\beta\right)^{2}} \sum_{k=0}^{\infty} \frac{p_{k}\left(q ;[n]_{q} t\right)}{[k]_{q} !}[k]_{q}^{2} \\
= & \left(x+\frac{[n]_{q} t}{[n]_{q}+\beta}\right)^{2}+\frac{2 x\left[\alpha+R D_{q}(A(1))\right]}{[n]_{q}+\beta} \\
& +\frac{\alpha^{2}+2 R \alpha D_{q}\left(A(1)+D_{q}^{2}(A(1))\right.}{\left([n]_{q}+\beta\right)^{2}}+\frac{[n]_{q} t\left[2 \alpha+D_{q}(A(1))\right]}{\left([n]_{q}+\beta\right)^{2}}+\frac{q[n]_{q} t}{\left([n]_{q}+\beta\right)^{2}} .
\end{aligned}
$$

Hence, the proof is completed.

Theorem 2.2. Assume that $q:=\left(q_{n}\right), 0<q_{n}<1$ be a sequence satisfying the following conditions:

$$
s t-\lim _{n} q_{n}=1, \quad s t-\lim _{n} q_{n}^{n}=b, \quad b<1
$$

Then, if $f$ is any monotone increasing continuous function defined on $[0, a]$, we have the following:

$$
s t-\lim _{n}\left\|T_{n, t}^{\alpha, \beta}\left(f, q_{n} ; .\right)-f\right\|_{C[0, a]}=0 .
$$

Proof. It is enough to prove that

$$
s t-\lim _{n}\left\|T_{n, t}^{\alpha, \beta}\left(e_{v}(t), q_{n} ; .\right)-e_{v}(t)\right\|_{C[0, a]}=0
$$

where $v=0,1,2$.

From the equation (2.1), it is easy to obtain that

$$
s t-\lim _{n}\left\|T_{n, t}^{\alpha, \beta}\left(e_{0}(t), q_{n} ; .\right)-e_{0}(t)\right\|_{C[0, a]}=0 .
$$

By taking $\sup _{x, t \in[0, a]}$ in (2.2), we get

$$
\left\|T_{n, t}^{\alpha, \beta}\left(e_{1}(t), q_{n} ; .\right)-e_{1}(t)\right\|_{C[0, a]} \leqslant \frac{\alpha+R D_{q}(A(1))}{[n]_{q}+\beta}+\frac{\beta a}{[n]_{q}+\beta} .
$$

Now, let $\epsilon>0$ be given, define the following sets:

$$
\begin{aligned}
K & :=\left\{k:\left\|T_{n, t}^{\alpha, \beta}\left(e_{1}(t), q_{k} ; .\right)-e_{1}(t)\right\| \geqslant \epsilon\right\}, \\
K_{1} & :=\left\{k: \frac{\beta a}{[n]_{q_{k}}+\beta} \geqslant \frac{\epsilon}{2}\right\}, \\
K_{2} & :=\left\{k: \frac{R D_{q}(A(1))+\alpha}{[n]_{q_{k}}+\beta} \geqslant \frac{\epsilon}{2}\right\},
\end{aligned}
$$

such that $K \subseteq K_{1} \cup K_{2}$.

From (2.7), one can see that

$$
\delta\left\{k \leqslant n:\left\|T_{n, t}^{\alpha, \beta}\left(e_{1}(t), q_{n} ; .\right)-e_{1}(t)\right\|_{C[0, a]} \geqslant \epsilon\right\} \leqslant \delta\left\{k \leqslant n: \frac{\beta a}{[n]_{q_{k}}+\beta} \geqslant \frac{\epsilon}{2}\right\}+\delta\left\{k \leqslant n: \frac{R+\alpha}{[n]_{q_{k}}+\beta} \geqslant \frac{\epsilon}{2}\right\} .
$$

By (2.7), it is obvious that,

$$
s t-\lim _{n}\left(\frac{1}{[n]_{q_{n}}+\beta}\right)=0 .
$$

Thus, we have

$$
s t-\lim _{n}\left\|T_{n, t}^{\alpha, \beta}\left(e_{1}(t), q_{n} ; .\right)-e_{1}\right\|_{C[0, a]}=0 .
$$


By taking $\sup _{x, t \in[0, a]}$ in (2.3), one can write the following

$$
\begin{aligned}
\left\|T_{n, t}^{\alpha, \beta}\left(e_{2}(t), q_{n} ; .\right)-e_{2}(t)\right\|_{C[0, a]} \leqslant & \frac{\beta^{2} a^{2}[n]_{q}}{[n]_{q}+\beta}+\frac{2 a\left[\alpha+R D_{q}(A(1))\right]}{[n]_{q}+\beta}+\frac{\alpha^{2}+2 R \alpha D_{q}(A(1))+D_{q}^{2}(A(1))}{\left([n]_{q}+\beta\right)^{2}} \\
& +\frac{[n]_{q} a\left[2 \alpha+D_{q}(A(1))\right]}{\left([n]_{q}+\beta\right)^{2}}+\frac{q[n]_{q} a}{\left([n]_{q}+\beta\right)^{2}} .
\end{aligned}
$$

It is obvious that

$$
s t-\lim _{n}\left(\frac{1}{\left([n]_{q_{n}}+\beta\right)^{2}}\right)=0, s t-\lim _{n}\left(\frac{[n]_{q_{n}}}{\left([n]_{q_{n}}+\beta\right)^{2}}\right)=0 \text { and } s t-\lim _{n}\left(\frac{q_{n}[n]_{q_{n}}}{\left([n]_{q_{n}}+\beta\right)^{2}}\right)=0 .
$$

Now, let $\epsilon>0$ be given, we define the following sets:

$$
\begin{aligned}
& V:=\left\{k:\left\|T_{n, t}^{\alpha, \beta}\left(e_{2}(t), q_{k} ; .\right)-e_{2}(t)\right\| \geqslant \epsilon\right\}, \\
& V_{1}:=\left\{k: \frac{2 a\left[\alpha+R D_{q}(A(1))\right]}{[n]_{q_{k}}+\beta} \geqslant \frac{\epsilon}{4}\right\}, \\
& V_{2}:=\left\{k: \frac{\alpha^{2}+2 R \alpha D_{q}\left((A(1))+D_{q}^{2}(A(1))+\beta^{2} a^{2}\right.}{\left([n]_{q_{k}}+\beta\right)^{2}} \geqslant \frac{\epsilon}{4}\right\}, \\
& V_{3}:=\left\{k: \frac{a[n]_{q_{k}}\left[2 \alpha+D_{q}(A(1))\right]}{\left([n]_{q_{k}}+\beta\right)^{2}} \geqslant \frac{\epsilon}{4}\right\}, \\
& V_{4}:=\left\{k: \frac{a q_{k}[n]_{q_{k}}}{\left([n]_{q_{k}}+\beta\right)^{2}} \geqslant \frac{\epsilon}{4}\right\}
\end{aligned}
$$

such that $V \subseteq V_{1} \cup V_{2} \cup V_{3} \cup V_{4}$.

Thus, we obtain

$$
\begin{aligned}
\delta\left\{k \leqslant n:\left\|T_{n, t}^{\alpha, \beta}\left(e_{2}(t), q_{n} ; .\right)-e_{2}(t)\right\|_{C[0, a]} \geqslant \epsilon\right\} & \leqslant \delta\left\{k \leqslant n: \frac{2 a\left[\alpha+D_{q}(A(1))\right]}{[n]_{q_{k}}+\beta} \geqslant \frac{\epsilon}{4}\right\} \\
& +\delta\left\{k \leqslant n: \frac{\alpha^{2}+2 R \alpha D_{q}\left((A(1))+D_{q}^{2}(A(1))+\beta^{2} a^{2}\right.}{\left([n]_{q_{k}}+\beta\right)^{2}} \geqslant \frac{\epsilon}{4}\right\} \\
& +\delta\left\{k \leqslant n: \frac{a[n]_{q_{k}}\left[2 \alpha+D_{q}(A(1))\right]}{\left([n]_{q_{k}}+\beta\right)^{2}} \geqslant \frac{\epsilon}{4}\right\} \\
& +\delta\left\{k \leqslant n: \frac{a q_{k}[n]_{q_{k}}}{\left([n]_{q_{k}}+\beta\right)^{2}} \geqslant \frac{\epsilon}{4}\right\} .
\end{aligned}
$$

Hence, (2.8), (22) and (2.10) imply that

$$
s t-\lim _{n}\left\|T_{n, t}^{\alpha, \beta}\left(e_{2}(t), q_{n} ; .\right)-e_{2}(t)\right\|_{C[0, a]}=0 .
$$

\section{RATES OF STATisticAl CONVERGENCE}

In this section, we give the rates of statistical convergence of the operators $T_{n, t}^{\alpha, \beta}(f ; q ; x)$ by means of modulus of continuity with the help of functions from Lipschitz class.

The modulus of continuity of $f, \omega(f, \delta)$ is defined by

$$
\omega(f, \delta)=\sup _{\substack{|x-y| \leqslant \delta \\ x, y \in[0, a]}}|f(x)-f(y)| .
$$

It is well-known that for a function $f \in C[0, a]$,

$$
\lim _{n \rightarrow 0^{+}} \omega(f, \delta)=0
$$

for any $\delta>0$

$$
|f(x)-f(y)| \leqslant \omega(f, \delta)\left(\frac{|x-y|}{\delta}+1\right) .
$$

Now, we prove the following theorem for the rate of pointwise convergence of the operators $T_{n, t}^{\alpha, \beta}(f ; q ; x)$ to the function $f(x+t)$ by means of modulus of continuity. 
Theorem 3.1. If the sequence $q:=\left(q_{n}\right)$ satisfies the condition 2.7), $x \in[0, \infty)$ and $t \geqslant 0$, then we have

$$
\left|T_{n, t}^{\alpha, \beta}(f ; q ; x)-f(x)\right| \leqslant 2 \omega\left(f, \sqrt{\delta_{n, t}}\right),
$$

for all $f \in C^{*}[0, \infty)$, where $\delta_{n, t}=T_{n, t}^{\alpha, \beta}\left(\left(s-e_{1}(t)\right)^{2} ; q_{n} ; x\right)$

Proof. To prove the theorem, we will use the linearity and positivity of the operators $T_{n, t}^{\alpha, \beta}(f ; q ; x)$. By (3.1), we have

$$
\begin{aligned}
\left|T_{n, t}^{\alpha, \beta}(f ; q ; x)-f(x+t)\right| & \leqslant \frac{E_{q}^{-[n]_{q} t}}{A(1)} \sum_{k=0}^{\infty} \frac{p_{k}\left(q ;[n]_{q} t\right)}{[k]_{q} !}\left|f\left(x+\frac{[k]_{q}+\alpha}{[n]_{q}+\beta}\right)-f(x+t)\right| \\
& \leqslant \frac{E_{q}^{-[n]_{q} t}}{A(1)} \sum_{k=0}^{\infty} \frac{p_{k}\left(q ;[n]_{q} t\right)}{[k]_{q} !} \omega(f, \delta)\left\{\frac{1}{\delta}\left|\frac{[k]_{q}+\alpha}{[n]_{q}+\beta}-t\right|+1\right\} \\
& =\left\{\frac{1}{\delta} \frac{E_{q}^{-[n]_{q} t}}{A(1)} \sum_{k=0}^{\infty} \frac{p_{k}\left(q ;[n]_{q} t\right)}{[k]_{q} !}\left|\frac{[k]_{q}+\alpha}{[n]_{q}+\beta}-t\right|+1\right\} \omega(f, \delta) .
\end{aligned}
$$

If we apply Cauchy-Schwarz inequality for sums, we obtain

$$
\sum_{k=0}^{\infty} \frac{p_{k}\left(q ;[n]_{q} t\right)}{[k]_{q} !}\left|\frac{[k]_{q}+\alpha}{[n]_{q}+\beta}-t\right|^{2} \leqslant\left(\sum_{k=0}^{\infty} \frac{p_{k}\left(q ;[n]_{q} t\right)}{[k]_{q} !}\right)^{1 / 2}\left(\sum_{k=0}^{\infty} \frac{p_{k}\left(q ;[n]_{q} t\right)}{[k]_{q} !}\left(\frac{[k]_{q}+\alpha}{[n]_{q}+\beta}-t\right)^{2}\right)^{1 / 2} .
$$

Using above inequality and by (2.1), we have

$$
\begin{aligned}
\left|T_{n, t}^{\alpha, \beta}(f ; q ; x)-f(x+t)\right| & \leqslant \omega(f, \delta)\left\{1+\frac{1}{\delta}\left[\frac{E_{q}^{-[n]_{q} t}}{A(1)} \sum_{k=0}^{\infty} \frac{p_{k}\left(q ;[n]_{q} t\right)}{[k]_{q} !}\left(\frac{[k]_{q}+\alpha}{[n]_{q}+\beta}-t\right)^{2}\right]^{1 / 2}\right\} \\
& =\omega(f, \delta)\left\{1+\frac{1}{\delta}\left[\frac{E_{q}^{-[n]_{q} t}}{A(1)} \sum_{k=0}^{\infty} \frac{p_{k}\left(q ;[n]_{q} t\right)}{[k]_{q} !}\left(\frac{[k]_{q}+\alpha}{[n]_{q}+\beta}-t\right)^{2}\right]^{1 / 2}\right\} \\
& \left.=\omega(f, \delta)\left\{1+\frac{1}{\delta}\left[T_{n, t}^{\alpha, \beta}\left(s-e_{1}(t)\right)^{2} ; q ; x\right)\right]^{1 / 2}\right\}
\end{aligned}
$$

such that we choose $\delta=\sqrt{\delta_{n, t}}=T_{n, t}^{\alpha, \beta}\left(\left(s-e_{1}(t)\right)^{2} ; q_{n} ; x\right)$. This step concludes the proof.

Theorem 3.2. If the sequence $q:=\left(q_{n}\right)$ satisfies the condition (2.7) and $f \in C[0, a]$, then we have

$$
\left\|T_{n, t}^{\alpha, \beta}\left(f ; q_{n} ; .\right)-f(.)\right\|_{C[0, a]} \leqslant 2 \omega\left(f, \sqrt{\delta_{n}}\right),
$$

where

$$
\delta_{n}=\frac{\beta^{2} t^{2}[n]_{q}}{\left([n]_{q}+\beta\right)^{2}}+\frac{\alpha^{2}+2 R \alpha D_{q}(A(1))+D_{q}^{2}(A(1))}{\left([n]_{q}+\beta\right)^{2}}+\frac{[n]_{q} t\left[2 \alpha+D_{q}(A(1))\right]}{\left([n]_{q}+\beta\right)^{2}}+\frac{q[n]_{q} t}{\left([n]_{q}+\beta\right)^{2}} .
$$

Proof. Now, let us estimate the second moment of the operators $T_{n, t}^{\alpha, \beta}(f ; q ; x)$. From (2.1)-(2.3), we get

$$
\begin{aligned}
T_{n, t}^{\alpha, \beta}\left(\left(s-e_{1}(t)\right)^{2} ; q ; x\right)= & \frac{t^{2}[n]_{q}^{2}}{\left([n]_{q}+\beta\right)^{2}}+\frac{\alpha^{2}+2 R \alpha D_{q}(A(1))+D_{q}^{2}(A(1))}{\left([n]_{q}+\beta\right)^{2}} \\
& +\frac{[n]_{q} t\left[2 \alpha+D_{q}(A(1))\right]}{\left([n]_{q}+\beta\right)^{2}}+\frac{q[n]_{q} t}{\left([n]_{q}+\beta\right)^{2}} .
\end{aligned}
$$

By Theorem 3.1 we have

$$
\left.\left|T_{n, t}^{\alpha, \beta}(f ; q ; x)-f(x+t)\right| \leqslant \omega(f, \delta)\left\{1+\frac{1}{\delta}\left[T_{n, t}^{\alpha, \beta}\left(s-e_{1}(t)\right)^{2} ; q ; x\right)\right]^{1 / 2}\right\}
$$

Substituting (3.2) into (3.3) and letting $\delta=\delta_{n}$ in (3.3), we obtain

$$
\left\|T_{n, t}^{\alpha, \beta}\left(f ; q_{n} ; .\right)-f(.)\right\|_{C[0, a]} \leqslant 2 \omega\left(f, \sqrt{\delta_{n}}\right) .
$$

Thus, we get the desired result. 
Now, we give the rate of convergence of the operators $T_{n, t}^{\alpha, \beta}$ with the help of functions from Lipschitz class $\operatorname{Lip}_{M}(\alpha)$ where $M>0$ and $0<\alpha \leqslant 1$. A function $f$ is an element of $\operatorname{Lip}_{M}(\alpha)$ if

$$
|f(x)-f(y)| \leqslant M|x-y|^{\alpha}(x, y \in[a, b]) .
$$

Theorem 3.3. Let $q:=\left(q_{n}\right)$ be a sequence satisfies the condition 2.7) and $f \in \operatorname{Lip}_{M}(\alpha), 0<\alpha \leqslant 1$, then we have

$$
\left|T_{n, t}^{\alpha, \beta}\left(f ; q_{n} ; .\right)-f(.)\right| \leqslant M \delta_{n}^{\alpha},
$$

where $\delta_{n}=\left(T_{n, t}^{\alpha, \beta}\left(e_{1}(t)-s\right)^{2}, q ; x\right)^{1 / 2}$.

Proof. Since $T_{n, t}^{\alpha, \beta}$ is linear and positive and by (3.4), we obtain

$$
\begin{aligned}
\left|T_{n, t}^{\alpha, \beta}\left(f ; q_{n} ; x\right)-f(x)\right| & \leqslant T_{n, t}^{\alpha, \beta}(|f(t)-f(x)|, q ; x) \\
& \leqslant M T_{n, t}^{\alpha, \beta}\left(|t-x|^{\alpha}, q ; x\right) .
\end{aligned}
$$

Assuming $p=\frac{1}{\alpha}, q=\frac{\alpha}{2-\alpha}$ and applying the Hölder inequality, we get

$$
\begin{aligned}
\left|T_{n, t}^{\alpha, \beta}\left(f ; q_{n} ; x\right)-f(x)\right| & \leqslant T_{n, t}^{\alpha, \beta}(|f(t)-f(x)|, q ; x) \\
& \left.\leqslant M\left\{T_{n, t}^{\alpha, \beta}\left(e_{1}-x\right)^{2}, q ; x\right)\right\}^{\alpha / 2} .
\end{aligned}
$$

Taking $\delta_{n, t}=\left(T_{n, t}^{\alpha, \beta}\left(e_{1}-s\right)^{2}, q ; x\right)^{1 / 2}$. We get the desired result.

\section{Local Approximation}

In this section, we state the local approximation theorem of the operators $T_{n, t}^{\alpha, \beta}(f ; q ; x)$. Let $C_{B}[0, \infty)$ be the space of all real valued continuous bounded functions $f$ on $[0, \infty)$ with the norm $\|f\|=\sup \{|f(x)|: x \in[0, \infty)\}$. The K-functional of $f$ is defined by

$$
K_{2}(f ; \delta)=\inf _{g \in W^{2}}\left\{\|f-g\|+\delta\left\|g^{\prime \prime}\right\|\right\},
$$

where $\delta>0$ and $W^{2}=\left\{g \in C_{B}[0, \infty): g^{\prime}, g^{\prime \prime} \in C_{B}[0, \infty)\right\}$. By Devore-Lorentz [1, p. 177], there exists an absolute constant $C>0$ such that

$$
K_{2}(f, \delta) \leqslant C \omega_{2}(f, \sqrt{\delta})
$$

where

$$
\omega_{2}(f, \sqrt{\delta})=\sup _{0<h \leqslant 0} \sup _{x \in[0, \infty)}|f(x+2 h)-2 f(x+h)+f(x)|
$$

is the second order modulus of smoothness of $f$. Moreover,

$$
\omega(f, \delta)=\sup _{0<h \leqslant 0} \sup _{x \in[0, \infty)}|f(x+h)-f(x)|
$$

denotes the modulus of continuity of $f$.

Now, we give the direct local approximation theorem for the operators $T_{n, t}^{\alpha, \beta}(f ; q ; x)$.

Theorem 4.1. Let $q \in(0,1)$. We have

$$
\left|T_{n, t}^{\alpha, \beta}(f ; q ; x)-f(x+t)\right| \leqslant C \omega_{2}\left(f, \delta_{n}\right)+\omega\left(f, \frac{\alpha+D_{q}(A(1)) R-\beta t}{[n]_{q}+\beta}\right)
$$

$\forall x \in[0, \infty), f \in C_{B}[0, \infty)$, where $C$ is a positive constant.

Proof. Let us define the following operators

$$
\widetilde{T}_{n, t}^{\alpha, \beta}(f ; q ; x)=T_{n, t}^{\alpha, \beta}(f ; q ; x)-f\left(x+\frac{[n]_{q} t+\alpha+D_{q}(A(1)) R}{[n]_{q}+\beta}\right)+f(x+t),
$$

$x \in[0, \infty)$. The operators $\widetilde{T}_{n, t}^{\alpha, \beta}(f ; q ; x)$ are linear. Thus, we have the following:

$$
\widetilde{T}_{n, t}^{\alpha, \beta}(s-(x+t) ; q ; x)=0,
$$

(see Lemma 2.1). Let $g \in W^{2}$, from Taylor's expansion

$$
g(s)=g(x+t)+g^{\prime}(x+t)(s-(x+t))+\int_{x+t}^{s}(s-u) g^{\prime \prime}(x) d u,
$$

$s \in[0, \infty)$ and (4.3) we obtain

$$
\widetilde{T}_{n, t}^{\alpha, \beta}(g ; q ; x)=g(x+t)+\widetilde{T}_{n, t}^{\alpha, \beta}\left(\int_{x+t}^{s}(s-u) g^{\prime \prime}(x) d u\right) .
$$


By (4.2), we have the following

$$
\begin{aligned}
\left|\widetilde{T}_{n, t}^{\alpha, \beta}(g ; q ; x)-g(x+t)\right| \leqslant & \left|T_{n, t}^{\alpha, \beta}\left(\int_{x+t}^{s}(s-u) g^{\prime \prime}(u) d u\right)\right| \\
& +\left|\int_{x+t}^{x+\frac{[n]_{q} t+\alpha+D_{q}(A(1)) R}{[n]_{q}+\beta}}\left(x+\frac{[n]_{q} t+\alpha+D_{q}(A(1)) R}{[n]_{q}+\beta}-u\right) g^{\prime \prime}(u) d u\right| \\
\leqslant & T_{n, t}^{\alpha, \beta}\left(\left|\int_{x+t}^{s}(s-u) g^{\prime \prime}(u) d u\right|, x\right)+\int_{x+t}^{x+\frac{[n]_{q} t+\alpha+D_{q}(A(1)) R}{[n]_{q}+\beta}}\left|x+\frac{[n]_{q} t+\alpha+R}{[n]_{q}+\beta}-u\right|\left|g^{\prime \prime}(u)\right| \\
\leqslant & \leqslant\left(T_{n, t}^{\alpha, \beta}(s-(x+t))^{2}+\left(x+\frac{[n]_{q} t+\alpha+D_{q}(A(1)) R}{[n]_{q}+\beta}\right)^{2}\right)\left\|g^{\prime \prime}\right\|
\end{aligned}
$$

Using (3.2), we get

$$
T_{n, t}^{\alpha, \beta}\left((s-(x+t))^{2} ; q ; x\right)+\left(x+\frac{[n]_{q} t+\alpha+D_{q}(A(1)) R}{[n]_{q}+\beta}\right)^{2} \leqslant \delta_{n}+\left(x+\frac{[n]_{q} t+\alpha+D_{q}(A(1)) R}{[n]_{q}+\beta}\right)^{2} .
$$

Thus, by (4.4), we obtain

$$
\left|\widetilde{T}_{n, t}^{\alpha, \beta}(g ; q ; x)-g(x+t)\right| \leqslant \delta_{n}+\left(x+\frac{[n]_{q} t+\alpha+D_{q}(A(1)) R}{[n]_{q}+\beta}\right)^{2} .
$$

By (1.1), (2.1) and (4.2), we get

$$
\begin{aligned}
\left|\widetilde{T}_{n, t}^{\alpha, \beta}(f ; q ; x)\right| & \leqslant T_{n, t}^{\alpha, \beta}(f ; q ; x)+2\|f\| \\
& \leqslant\|f\| T_{n, t}^{\alpha, \beta}(1 ; q ; x)+2\|f\| \\
& \leqslant 3\|f\| .
\end{aligned}
$$

Now, by (4.2), (4.5) and (4.6)

$$
\begin{aligned}
\left|T_{n, t}^{\alpha, \beta}(f ; q ; x)-f(x+t)\right| \leqslant & \left|\widetilde{T}_{n, t}^{\alpha, \beta}(f-g ; q ; x)-(f-g)(x+t)\right|+\left|\widetilde{T}_{n, t}^{\alpha, \beta}(g ; q ; x)-g(x+t)\right| \\
& +\left|f\left(x+\frac{[n]_{q} t+\alpha+D_{q}(A(1)) R}{[n]_{q}+\beta}\right)-f(x+t)\right| \\
\leqslant & \left.4\|f-g\|+\delta_{n}\right)\left\|g^{\prime \prime}\right\|
\end{aligned}
$$

In view of (4.1), $\forall q \in(0,1)$ we get

$$
\left|T_{n, t}^{\alpha, \beta}(f ; q ; x)-f(x+t)\right| \leqslant C \omega_{2}\left(f, \delta_{n}\right)+\omega\left(\frac{\alpha+D_{q}(A(1)) R-\beta t}{[n]_{q}+\beta}\right)
$$

and this concludes the proof.

\section{REFERENCES}

[1] R.A. DeVore, G.G. Lorentz, Constructive Approximation, Springer, Berlin, 1993.

[2] I. Büyükyazıcı, H. Tanberkan, S. K. Serenbay, C. Atakut, Approximation by Chlodowsky type JakimovskiLeviatan operators, Journal of Computational and Applied Mathematics 259 (2014), 153-163.

[3] I. Büyükyazıcı, C. Atakut, On Stancu type generalization of $q$-Baskakov operators, Mathematical and Computer Modelling 52(5) (2010), 752-759.

[4] A. Aral, V. Gupta, On the Durrmeyer type modification of the $q$-Baskakov type operators, Nonlinear Analysis: Theory, Methods \& Applications 72(3) (2010), 1171-1180.

[5] C. Atakut, I. Buyukyazici, The rate of convergence of the q-analogue of Favard Szàsz type operators, Journal of Computational Analysis and Applications,Vol. 13, No.4, (2011) 673-682.

[6] C. Atakut, I. Buyukyazici, Stancu type generalization of the Favard Szàsz operators, Applied Mathematics Letters, 23 (2010), 1479-1482.

[7] A. Aral, V. Gupta, R. P. Agarwal, Applications of q-calculus in operator theory, Berlin, Springer, 2013.

[8] A. Ciupa, On a generalized Favard Szàsz type operator, Seminar on Numerical and statistical calculus, Univ. Babeş-Bolyai Cluj-Napoca, preprint nr.1 (1994), 3338.

[9] D.D. Stancu, A study of the remainder in an approximation formula using a Favard Szàsz type operator, Stud. Univ. Babes-Bolyai, Math. XXV (1980), 7076.

[10] A. Jakimovski, D. Leviatan, Generalized Szàsz operators for the approximation in the infinite interval, Mathematica (Cluj), 34, 1969, 97-103.

[11] H. Fast, Sur la convergence statistique, Colloq. Math. Studia Math. 2 (1951) 241-244. 
[12] A.D. Gadjiev, C. Orhan, Some approximation properties via statistical convergence, Rocky Mountain J. Math. 32 (2002) 129-138.

[13] Ö. Dalmanoğlu, O. Doğru, On statistical approximation properties of Kantorovich type $q$-Bernstein operators, Mathematical and Computer Modelling 52(2010), 760-771.

[14] C. Radu, Statistical approximation properties of Kantorovich operators based on q-integers, Creative Math. and Inf, 17 (2008), 75-84.

[15] O. Doğru, Kadir Kanat, On statistical approximation properties of the Kantorovich type Lupaş operators, Mathematical and Computer Modelling, 55(3) (2012), 1610-1621.

[16] P. N. Agrawal, V. Gupta, A. Sathish Kumar, A. Kajla, Generalized Baskakov-Szasz type operators, Applied Mathematics and Computation, 236 (2014), 311-324.

[17] O.H.H. Edely, M. Mursaleen, A. Khan, Approximation for periodic functions via weighted statistical convergence, Applied Mathematics and Computation, 219 (2013), 8231-8236.

(A. Karaisa) DEPARTMENT OF MATHEMATICS-COMPUTER SCIENCE, FACULTY OF SCIENCES, NECMETTIN ERBAKAN UNIVERSITY, MERAM YERLEŞKESI , 42090 MERAM, KONYA, TURKEY

E-mail address, A. Karaisa: alikaraisa@hotmail.com, akaraisa@konya.edu.tr

(D.T. Tollu) DEPARTMENT OF MATHEMATICS-COMPUTER SCIENCE, FACULTY OF SCIENCES, NECMETTIN

ERBAKAN UNIVERSITY, MERAM YERLEŞKESI , 42090 MERAM, KONYA, TURKEY

E-mail address, D.T. Tollu: dttollu@konya.edu.tr

(Y. Asar) DEPARTMENT OF MATHEMATICS-COMPUTER SCIENCE, FACULTY OF SCIENCES, NECMETTIN

ERBAKAN UNIVERSITY, MERAM YERLEŞKESI , 42090 MERAM, KONYA, TURKEY

E-mail address, Y. Asar: yasar@konya.edu.tr 